\title{
Strongly Positive Semigroups and Faithful Invariant States
}

\author{
Derek W. Robinson ${ }^{\star}$ \\ School of Mathematics, University of New South Wales, Australia and Department of Mathematics, \\ Bedford College, London University, London, England
}

\begin{abstract}
Let $(\mathscr{M}, \tau, \omega)$ denote a $W^{*}$-algebra $\mathscr{M}$, a semigroup $t>0 \mapsto \tau_{t}$ of linear maps of $\mathscr{M}$ into $\mathscr{M}$, and a faithful $\tau$-invariant normal state $\omega$ over $\mathscr{M}$. We
\end{abstract} assume that $\tau$ is strongly positive in the sense that

$$
\tau_{t}\left(A^{*} A\right) \geqq \tau_{t}(A)^{*} \tau_{t}(A)
$$

for all $A \in \mathscr{M}$ and $t>0$. Therefore one can define a contraction semigroup $T$ on $\mathscr{H}=\bar{M} \Omega$ by

$$
T_{t} A \Omega=\tau_{t}(A) \Omega, \quad A \in \mathscr{M},
$$

where $\Omega$ is the cyclic and separating vector associated with $\omega$. We prove

1. the fixed points $\mathscr{M}(\tau)$ of $\tau$ are given by $\mathscr{M}(\tau)=\mathscr{M} \cap T^{\prime}=\mathscr{M} \cap E^{\prime}$, where $E$ is the orthogonal projection onto the subspace of $T$-invariant vectors,

2. the state $\omega$ has a unique decomposition into $\tau$-ergodic states if, and only if, $\mathscr{M}(\tau)$ or $\{\mathscr{M} \cup E\}^{\prime}$ is abelian or, equivalently, if $(\mathscr{M}, \tau, \omega)$ is $\mathbb{R}_{+}$-abelian,

3. the state $\omega$ is $\tau$-ergodic if, and only if, $\mathscr{M} \cup E$ is irreducible or if

$$
\inf _{\omega^{\prime \prime} \in \operatorname{Co} \omega^{\prime} \circ \tau}\left\|\omega^{\prime \prime}-\omega\right\|=0
$$

for all normal states $\omega^{\prime}$ where Co $\omega^{\prime} \circ \tau$ denotes the convex hull of $\left\{\omega^{\prime} \circ \tau_{t}\right\}_{t>0}$.

Subsequently we assume that $\tau$ is 2-positive, $T$ is normal, and $T_{t}^{*} \mathscr{M}_{+} \Omega \subseteq \overline{\mathscr{M}_{+} \Omega}$, and then prove

4. there exists a strongly positive semigroup $|\tau|$ which commutes with $\tau$ and is determined by

$$
|\tau|_{t}(A) \Omega=\left|T_{t}\right| A \Omega,
$$

5. results similar to 1 and 2 apply to $|\tau|$ but the $\tau$-invariant state $\omega$ is $|\tau|$ ergodic if, and only if,

for all normal states $\omega^{\prime}$.

$$
\lim _{t \rightarrow \infty}\left\|\omega^{\prime} \circ \tau_{t}-\omega\right\|=0
$$

* New address: Department of Mathematics, Institute of Advanced Studies, Australian National University, Canberra 


\section{Preliminaries}

The aims of this paper, and its notation are described in the abstract. Throughout we adopt the assumptions outlined in the first paragraph of the abstract. Our results are an extension of the well established theory of states invariant under a group of *-automorphisms (see, for example [1, Chap. 4]) and also a generalization of work by Frigerio [2] in the semigroup case. We begin with some preliminary observations which are subsequently useful for characterizing the fixed point set $\mathscr{M}(\tau)$.

First note that as $\tau$ is positive it is automatically self-adjoint, i.e., $\tau_{t}(A)^{*}=\tau_{t}\left(A^{*}\right)$ for all $A \in \mathscr{M}$. Further as $\tau$ is strongly positive, and $\omega$ is $\tau$-invariant, the operators $T_{t}$ are well-defined linear contractions, and since $\omega$ is faithful $\tau_{t}(\mathbb{1})=\mathbb{1}$.

Second define the $\mathscr{M}$-valued sesquilinear forms $S_{t}$ by

$$
S_{t}(A, B)=\tau_{t}\left(A^{*} B\right)-\tau_{t}(A)^{*} \tau_{t}(B) .
$$

If $B \in \mathscr{M}(\tau)$ it follows by $\tau$-invariance of $\omega$ that

$$
\omega\left(S_{t}(B, B)\right)=0 \text {. }
$$

Since $S_{t}(B, B)$ is positive, and $\omega$ is faithful one concludes that $S_{t}(B, B)=0$. It then follows from the Cauchy-Schwarz inequality applied to $S_{t}$ in an arbitrary state over $\mathscr{M}$ that

$$
S_{t}(A, B)=0=S_{t}(B, A)
$$

for all $A \in \mathscr{M}$. (These observations are taken from $[2,3]$.)

Third let $C_{\lambda}(T)$ denote convex combinations of $T$,

$$
C_{\lambda}(t)=\sum_{i=1}^{n} \lambda_{i} T_{t_{i}}
$$

and $C_{\lambda}(\tau(A))$ the corresponding combinations of $\tau(A)$, i.e.,

$$
C_{\lambda}(\tau(A))=\sum_{i=1}^{n} \lambda_{i} \tau_{t_{i}}(A) .
$$

It follows from the Alaoglu-Birkhoff version of the mean ergodic theorem (see [1, Proposition 4.3.4]) that the net $C_{\lambda}(T)$ converges strongly to $E$. It then follows from the cyclicity of $\Omega$ for $\mathscr{M}^{\prime}$ that the corresponding net $C_{\lambda}(\tau(A))$ converges strongly to an $M_{\tau}(A) \in \mathscr{M}$, for each $A \in \mathscr{M}$. This mean value $M_{\tau}(A)$ of $A$ then satisfies

$$
M_{\tau}(A) A^{\prime} \Omega=A^{\prime} E A \Omega
$$

for all $A^{\prime} \in \mathscr{M}^{\prime}$. Moreover $M_{\tau}(A) \in \mathscr{M}(\tau)$. But conversely if $A \in \mathscr{M}(\tau)$ then $M_{\tau}(A)$ $=A \in \mathscr{M}(\tau)$. Thus the fixed point set $\mathscr{M}(\tau)$ is equal to the range $R\left(M_{\tau}\right)$ of the map $M_{\tau}$. Finally it follows from the action $\left({ }^{*}\right)$ that $\Omega$ is cyclic for $\mathscr{M}(\tau)$ in $E \mathscr{H}$.

\section{Invariant Elements}

The first result describes a variety of properties of the fixed point set.

Theorem 1. It follows that

$$
T_{t} A E=\tau_{t}(A) E
$$


for all $A \in \mathscr{M}$ and hence

$$
M_{\tau}(A) E=E A E .
$$

Moreover

$$
\mathscr{M}(\tau)=\mathscr{M} \cap E^{\prime}=\mathscr{M} \cap T^{\prime} .
$$

Proof. If $A^{*} \in \mathscr{M}$ and $B \in \mathscr{M}(\tau)$ then $S_{t}\left(A^{*}, B\right)=0$ by the discussion in the preliminary section. Hence applying $S_{t}\left(A^{*}, B\right)$ to $\Omega$ one has

$$
\tau_{t}(A B) \Omega=\tau_{t}(A) B \Omega
$$

or, equivalently

$$
T_{t} A B \Omega=\tau_{t}(A) B \Omega .
$$

But $B \Omega=M_{\tau}(B) \Omega=E B \Omega$ and $\Omega$ is cyclic for $\mathscr{M}(\tau)$ in $E \mathscr{K}$. Therefore the first statement of the theorem is valid. The second statement follows by taking mean values.

Next one also has $S_{t}\left(B^{*}, A\right)=0$ and applying this to $\Omega$ gives

$$
\tau_{t}(B A) \Omega=B \tau_{t}(A) \Omega
$$

or, equivalently

$$
T_{t} B A \Omega=B T_{t} A \Omega .
$$

Therefore $B \in T^{\prime}$ and consequently $\mathscr{M}(\tau) \subseteq \mathscr{M} \cap T^{\prime}$. But $\mathscr{M} \cap T^{\prime} \subseteq \mathscr{M} \cap E^{\prime}$ by the mean ergodic theorem. Finally if $B \in \mathscr{M} \cap E^{\prime}$ then

$$
B \Omega=E B \Omega=M_{\tau}(B) \Omega
$$

and $M_{\tau}(B)=B$ because $\Omega$ is separating. Thus $B \in \mathscr{M}(\tau)$ and equality of the three sets $\mathscr{M}(\tau), \mathscr{M} \cap T^{\prime}$, and $\mathscr{M} \cap E^{\prime}$ follows immediately.

Remark. It is not a priori evident that $\mathscr{M}(\tau)$ is a $W^{*}$-algebra. This was established in the present context by Frigerio [2]. It is also not evident that $\mathscr{M} \cap T^{\prime}$ is selfadjoint, since $T$ is not necessarily self-adjoint. This follows, however, from the identification with $\mathscr{M} \cap E^{\prime}$.

\section{Decomposition Theory}

Next we describe criteria for $\omega$ to have a unique decomposition into $\tau$-ergodic states, i.e., states extremal in the convex subset $E_{\mathscr{M}}^{\tau}$ of $\tau$-invariant states. The decomposition theory of states $\omega$ (see, for example, [1, Chap. 4]) has been developed in two parts. First one constructs measures $\mu$ on the state space $E_{\mathscr{M}}$ which are maximal with respect to the Choquet order and which have $\omega$ as barycentre. Second one derives conditions which ensure that $\mu$ is supported by the extremal points of the appropriate convex set. For this latter point separability of $\mathscr{H}$ is usually sufficient [1, Sect. 4.4]. Hence we concentrate on criteria for a unique maximal measure.

First recall that the general theory of measures on $E_{\mathcal{M}}$ with barycentre $\omega$ establishes a one-to-one correspondence between orthogonal measures $\mu$, abelian von Neumann subalgebras $\mathscr{B} \subseteq \mathscr{M}^{\prime}$, and orthogonal projections $P$ such that $P \Omega=\Omega$ and $P \mathscr{M} P \subseteq\{P \mathscr{M} P\}^{\prime}$. This correspondence is then extended to states 
invariant under a group of *automorphisms [1, Sect. 4.3] by incorporation of invariance properties into each of the sets. A similar generalization can be made for states $\omega$ invariant under a strongly positive semigroup $\tau$.

Proposition 1. There exists a one-to-one correspondence between the following;

1. Orthogonal measures $\mu$ with barycentre $\omega$ satisfying

$$
\mu\left(\widehat{\tau_{t}\left(A_{1}\right)} \hat{A}_{2}\right)=\mu\left(\hat{A}_{1} \hat{A}_{2}\right)
$$

for all $A_{1}, A_{2} \in \mathscr{M}$ and $t>0$ where $\hat{A}$ is given by $\hat{A} ; \omega^{\prime} \in E_{\mathscr{M}} \rightarrow \hat{A}\left(\omega^{\prime}\right)=\omega^{\prime}(A)$,

2. Abelian von Neumann subalgebras $\mathscr{B} \cong\{\mathscr{M} \cup E\}^{\prime}$,

3. Orthogonal projections $P$ on $\mathscr{H}$ such that

$$
P \Omega=\Omega, \quad P \leqq E, \quad P \mathscr{M} P \cong\{P \mathscr{M} P\}^{\prime} .
$$

Moreover the support of such $\mu$ is contained in the weak*-compact subset $E_{\mathscr{M}}^{\tau}$ of $\tau$-invariant states. Finally there exists a unique maximal measure $\mu$ on $E_{\mathscr{M}}^{\tau}$ with barycentre $\omega$ if, and only if, $\{\mathscr{M} \cup E\}^{\prime}$ is abelian and in this case $\mu$ is the orthogonal measure corresponding to $\{\mathscr{M} \cup E\}^{\prime}$.

Remark. For this result it is not necessary to assume that $\omega$ is faithful.

Proof. The proof of correspondence is basically a rearrangement of the arguments used in Sect. 4.3 of [1] for automorphism groups.

Let $\mu, \mathscr{B}, P$ denote the orthogonal measure, abelian subalgebra of $\mathscr{M}^{\prime}$, and the projection $P=[\mathscr{B} \Omega]$, which are associated with $\omega$ and which are in correspondence through the correspondences described in Theorem 4.1.25 of [1]. Now consider the three conditions.

$1 \Rightarrow 3$. From Condition 1 it follows that

$$
\begin{aligned}
\left(\Omega, A_{1} T_{t}^{*} P A_{2} \Omega\right) & \left.=\mu\left(\widehat{\left(\tau _ { t } \left(A_{1}\right.\right.}\right)\left(\widehat{A_{2}}\right)\right) \\
& =\mu\left(\hat{A}_{1} \hat{A}_{2}\right)=\left(\Omega, A_{1} P A_{2} \Omega\right) .
\end{aligned}
$$

Thus $T_{t}^{*} P=P$ and $E P=P=P E$.

$3 \Rightarrow 1$. This follows from the above equation.

$1 \Rightarrow 2$. Since $\mu$ is in correspondence with a $P$ such that $T_{t} P=P$ it follows by approximation that $\mu$ is supported by $E_{\mathscr{M}}^{\tau}$. Explicitly one chooses $\mathscr{N} \cong \mathscr{B}$ to be any finite-dimensional von Neumann algebra and denotes by $\mu_{\mathcal{N}}$ and $P_{\mathscr{N}}$ the corresponding orthogonal measure and projection. Since $\mathscr{N} \subseteq \mathscr{B}$ it follows that $P_{\mathscr{N}}$ $\leqq P$ and hence $P_{\mathscr{N}} \leqq E$. Therefore it follows from the proof of Proposition 4.3.2 of [1] that $\mu_{\mathscr{N}}$ is supported by $E_{\mathscr{M}}^{\tau}$. Furthermore since $\mathscr{N}$ is finite-dimensional $\mu_{\mathscr{N}}$ has finite support.

One now calculates

$$
\begin{aligned}
\left(\Omega, A T_{t} \kappa(f) B \Omega\right) & =\left(\Omega, \tau_{t}(A) B \kappa\left(f^{\prime}\right) \Omega\right) \\
& =\int d \mu_{\mathscr{N}}\left(\omega^{\prime}\right) f\left(\omega^{\prime}\right) \omega^{\prime}\left(\tau_{t}(A) B\right) \\
& =\int d \mu_{\mathscr{N}}\left(\omega^{\prime}\right) f\left(\omega^{\prime}\right)\left(\Omega^{\prime}, A T_{t}^{*} B \Omega^{\prime}\right),
\end{aligned}
$$


where the invariance of $\omega^{\prime}$ was used to introduce $T^{\prime}$ and $\kappa(f)$ is defined for $f \in L^{\infty}\left(\mu_{\mathscr{N}}\right)$ by

$$
(\Omega, A \kappa(f) B \Omega)=\int d \mu_{\mathscr{N}}\left(\omega^{\prime}\right) f\left(\omega^{\prime}\right) \omega^{\prime}(A B) .
$$

Now since $\mu_{\mathscr{N}}$ has finite support one obtains

$$
(\Omega, A E \kappa(f) B \Omega)=\int d \mu_{\mathscr{N}}\left(\omega^{\prime}\right) f\left(\omega^{\prime}\right)\left(\Omega^{\prime}, A E^{\prime} B \Omega^{\prime}\right)
$$

by taking mean values of $(*)$. Similarly

$$
\left(\Omega, A \kappa(f) T_{t} B \Omega\right)=\int d \mu_{\mathscr{N}}\left(\omega^{\prime}\right) f\left(\omega^{\prime}\right)\left(\Omega^{\prime}, A T_{t}^{\prime} B \Omega^{\prime}\right)
$$

and by mean values

$$
\begin{aligned}
(\Omega, A \kappa(f) E B \Omega) & =\int d \mu_{\mathscr{N}}\left(\omega^{\prime}\right) f\left(\omega^{\prime}\right)\left(\Omega^{\prime}, A E^{\prime} B \Omega^{\prime}\right) \\
& =(\Omega, A E \kappa(f) B \Omega) .
\end{aligned}
$$

Thus $\kappa(f) \supseteqq E^{\prime}$ and as $\mathscr{N}=\left\{\kappa(f) ; f \in L^{\infty}\left(\mu_{\mathscr{N}}\right)\right\}$ it follows that $\mathscr{N} \supseteq\{\mathscr{M} \cup E\}^{\prime}$. Finally it follows $\overline{\bar{b} y}$ limiting (see Lemma 4.1 .26 of [1]) that $\mathscr{B} \supset\{\mathscr{M} \cup \overline{\bar{E}}\}^{\prime}$.

$2 \Rightarrow 3$. This is evident.

Since $\mu_{\mathcal{N}}$ has finite support in $E_{\mathscr{M}}^{\tau}$ and since $\mu_{\mathscr{N}} \rightarrow \mu$ vaguely as one takes the limit over the net of finite-dimensional matrix subalgebras of $\mathscr{B}$ (see Lemma 4.1.26 of [1]) it follows that the support of $\mu$ is contained in $E_{\mathscr{M}}^{\tau}$.

The final statement concerning maximal measures and maximal abelian algebras follows by the argument used to prove Proposition 4.3.3 of [1] modified as in the above proof that $1 \Rightarrow 2$.

Although Proposition 1 is valid for any invariant $\omega$ more elaborate criteria for uniqueness are obtained if $\omega$ is also faithful.

Theorem 2. The following conditions are equivalent;

1. There exists a unique maximal measure $\mu$ on $E_{\mathcal{M}}^{\tau}$ with barycentre $\omega$,

2. The commutant $\{\mathscr{M} \cup E\}^{\prime}$ is abelian,

3. The fixed point algebra $\mathscr{M}(\tau)$ is abelian,

4. The set EME is abelian,

5 .

$$
\inf _{A^{\prime} \in \operatorname{Co} \tau(A)}\left|\omega^{\prime}\left(\left[A^{\prime}, B\right]\right)\right|=0
$$

for all $A, B \in \mathscr{M}$ and all $\tau$-invariant vector states $\omega^{\prime}$ of $\omega$, where $\operatorname{Co} \tau(A)$ denotes the convex hull of $\left\{\tau_{t}(A) ; t>0\right\}$.

If these conditions are satisfied, $\mu$ is the orthogonal measure corresponding to $E$ and $\{\mathscr{M} \cup E\}^{\prime}$.

Proof. $1 \Leftrightarrow 2$. This is the general criterion stated above.

$2 \Leftrightarrow 3$. Let $J$ be the modular conjugation associated with the pair $(\mathscr{M}, \Omega)$. It follows from Lemma 2 of [4] that $J$ and $E$ commute. Thus $J E J=E$ and

$$
J\{\mathscr{M} \cup E\}^{\prime} J=\left\{\mathscr{M}^{\prime} \cup E\right\}^{\prime}=\mathscr{M} \cap E^{\prime}=\mathscr{M}(\tau)
$$

and Conditions 2 and 3 are simultaneously valid.

$2 \Rightarrow 4$. The general correspondence theorem for orthogonal measures places the abelian algebra $\{\mathscr{M} \cup E\}^{\prime}$ in correspondence with the projection $P=\left[\{\mathscr{M} \cup E\}^{\prime} \Omega\right]$ 
and this projection then has the property that $P \mathscr{M} P$ is abelian. But $\Omega$ is cyclic for $\mathscr{M} \cap E^{\prime}$ in $E \mathscr{H}$ and $J$ commutes with $E$. Thus $\Omega$ is cyclic for $\mathscr{M}^{\prime} \cap E^{\prime}$ in $E \mathscr{H}$ and hence $P=E$.

$4 \Rightarrow 2$. This follows from the general correspondence theorem for orthogonal measures.

$4 \Leftrightarrow 5$. This follows by repetition of the argument used to prove the analogous statement in Proposition 4.3.7 of [1]. This argument has two essential ingredients, the mean ergodic theorem, and the relation

But the latter is valid by Theorem 1 .

$$
T_{t} A E=\tau_{t}(A) E .
$$

Remark. Condition 5 corresponds to $\mathbb{R}_{+}$-abelianness of $(\mathscr{M}, \tau, \omega)$ in the terminology used for automorphism groups. It is implied by the condition

$$
\lim _{t \rightarrow \infty} \omega^{\prime}\left(\left[\tau_{t}(A), B\right]\right)=0
$$

of asymptotic abelianness. This latter condition should correspond to a form of dispersion of the dynamical system $(\mathscr{M}, \tau)$ in the state $\omega$.

The foregoing arguments also provide characterizations of faithful $\tau$-ergodic states. If $\omega$ is $\tau$-ergodic then there is certainly a unique maximal measure $\mu$ with barycentre $\omega$ and $\mu=\delta_{\omega}$. But the point measure $\delta_{\omega}$ is an orthogonal measure in correspondence with the projection $E_{\Omega}$ on $\Omega$ and the abelian algebra $\mathbb{C} \mathbb{1}$. Thus $E=E_{\Omega}$ and $\{\mathscr{M} \cup E\}^{\prime}=\mathbb{C} \mathbb{1}$ by the last statement of Theorem 2. Consequently one has the following;

Theorem 3. The following conditions are equivalent;

1. $\omega$ is $\tau$-ergodic,

2. $\mathscr{M} \cup E$ is irreducible,

3. $\mathscr{M}(\tau)=\mathbb{C} \mathbb{1}$,

4. E has rank one.

The equivalences $1 \Leftrightarrow 3 \Leftrightarrow 4$ have previously been given by Frigerio [2] with some extraneous continuity assumptions on $\tau$. Frigerio also discusses other criteria of irreducibility. A similar result with faithfulness of $\omega$ replaced by a condition of asymptotic abelianness has been given in [5].

\section{Cluster Properties}

It follows from the mean ergodic theorem and the definition of the mean values $M_{\tau}(A)$ that

$$
\inf _{A^{\prime} \in \operatorname{Co} \tau(A)}\left|\omega^{\prime}\left(A^{\prime}\right)-\omega^{\prime}\left(M_{\tau}(A)\right)\right|=0
$$

for all normal states $\omega^{\prime}$. Moreover $\omega^{\prime} \circ M_{\tau}=\omega$ for all normal $\omega^{\prime}$ if, and only if, $E$ has rank one. Thus $\omega$ is $\tau$-ergodic if, and only if,

$$
\inf _{A^{\prime} \in \operatorname{Co} \tau(A)}\left|\omega^{\prime}\left(A^{\prime}\right)-\omega(A)\right|=0
$$

for all $A \in \mathscr{M}$ and all normal $\omega^{\prime}$. But these statements are also true in the uniform topology. 
Theorem 4. For every normal state $\omega^{\prime}$ one has

$$
\inf _{\omega^{\prime \prime} \in \operatorname{Co} \omega^{\prime} \circ \tau}\left\|\omega^{\prime \prime}-\omega^{\prime} \circ M_{\tau}\right\|=0 .
$$

Thus $\omega$ is $\tau$-ergodic if, and only if,

for all normal $\omega^{\prime}$.

$$
\inf _{\omega^{\prime \prime} \in \operatorname{Co} \omega^{\prime} \circ \tau}\left\|\omega^{\prime \prime}-\omega\right\|=0
$$

Proof. Each normal $\omega^{\prime}$ can be uniformly approximated by a finite linear combination of vector states $(\phi, A \phi)$. But since $\omega$ is faithful each $\phi$ can be strongly approximated by a vector of the form $A^{\prime} \Omega$ with $A^{\prime} \in \mathscr{M}^{\prime}$. It follows that each normal $\omega^{\prime}$ can be uniformly approximated by a state $l_{\psi}(A)=(\psi, A \Omega)$ with $\psi \in \mathscr{M}_{+}^{\prime} \Omega$. But

and hence

$$
\sum_{i=1}^{n} \lambda_{i} l_{\psi}\left(\tau_{t_{i}}(A)\right)-l_{\psi}\left(M_{\tau}(A)\right)=\left(\left(\sum_{i=1}^{n} \lambda_{i} T_{t_{i}}^{*}-E\right) \psi, A \Omega\right)
$$

$$
\left\|\sum_{i=1}^{n} \lambda_{i} l_{\psi} \circ \tau_{t_{i}}-l_{\psi} \circ M_{\tau}\right\| \leqq\left\|\left(\sum_{i=1}^{n} \lambda_{i} T_{t_{l}}^{*}-E\right) \psi\right\| .
$$

The first statement of the theorem now follows from the mean ergodic theorem and the second statement follows from the first.

Remark. Theorem 4 is also valid for a group of *automorphisms and it has an analogue for non-faithful states (see [6, Proposition 4]). Let $\omega$ be a general $\tau$-invariant state and suppose $\omega^{\prime} \leqq \lambda \omega$ for some $\lambda>0$. Thus $\omega^{\prime}(A)=\left(B^{\prime} \Omega, A \Omega\right)$ for some $B^{\prime} \in \mathscr{M}_{+}^{\prime}$. One can define the mean $\omega^{\prime} \circ M_{\tau}$ of $\omega^{\prime}$ by $\omega^{\prime} \circ M_{\tau}(A)=\left(B^{\prime} \Omega, E A \Omega\right)$ and then

$$
\inf _{\omega^{\prime \prime} \in \operatorname{Co} \omega^{\prime} \circ \tau}\left\|\omega^{\prime \prime}-\omega^{\prime} \circ M_{\tau}\right\|=0
$$

by the argument used to prove Theorem 4 . This result then extends to all states in the norm closure $S_{\omega}$ of the set $\left\{\omega^{\prime} ; \omega^{\prime} \leqq \lambda \omega\right\}$. But $S_{\omega}$ is equal to the set of normal states if and only if $\omega$ is faithful.

Theorem 4 demonstrates that for the convergence of means of normal states there is no distinction between strongly positive semigroups and groups of *-automorphisms, or between weak*-convergence and uniform convergence. Distinctions arise, however, if one considers pointwise limits as $t \rightarrow \infty$. For example if $\tau$ extends to a group of *automorphisms then it is automatically isometric and

$$
\left\|l_{\psi} \circ \tau_{t}-l_{\psi} \circ M_{\tau}\right\|=\sup _{\|A\|=1} \mid l_{\psi}\left(\tau_{t}(A)-l_{\psi}\left(M_{\tau}(A)\right)\left|=\sup _{\|A\|=1}\right|((\mathbb{1}-E) \psi, A \Omega) \mid .\right.
$$

Thus $\left\|\omega^{\prime} \circ \tau_{t}-\omega^{\prime} \circ M_{\tau}\right\|$ is independent of $t$ for all normal states $\omega^{\prime}$ and pointwise convergence occurs only in trivial cases. Non-trivial results are possible, however, for semigroups. The following gives a simple illustration which is subsequently useful.

Proposition 2. Assume that the semigroup $T$ associated with $\tau$ is self-adjoint. It follows that

$$
\lim _{t \rightarrow \infty}\left\|\omega^{\prime} \circ \tau_{t}-\omega^{\prime} \circ M_{\tau}\right\|=0
$$


for all normal states $\omega^{\prime}$ and hence $\omega$ is $\tau$-ergodic if, and only if,

$$
\lim _{t \rightarrow \infty}\left\|\omega^{\prime} \circ \tau_{t}-\omega\right\|=0
$$

for all $\omega^{\prime}$.

Proof. If one approximates $\omega^{\prime}$ by $l_{\psi}$ as in the proof of Theorem 4 one then obtains the estimate

$$
\left\|l_{\psi} \circ \tau_{t}-l_{\psi} \circ M_{\tau}\right\|^{2} \leqq\left\|\left(T_{t}-E\right) \psi\right\|^{2} .
$$

But for $A \in \mathscr{M}$ and $s<t$

$$
\begin{aligned}
\left(A \Omega, T_{2 t} A \Omega\right) & =\omega\left(\tau_{t}(A)^{*} \tau_{t}(A)\right) \\
& =\omega\left(\tau_{t-s}\left(\tau_{s}(A)\right)^{*} \tau_{t-s}\left(\tau_{s}(A)\right)\right) \\
& \leqq \omega\left(\tau_{s}(A)^{*} \tau_{s}(A)\right)=\left(A \Omega, T_{2 s} A \Omega\right) .
\end{aligned}
$$

Thus by standard monotonicity and density arguments $T_{t}$ converges strongly to $E$ and the first statement of the proposition is valid. Again the second statement follows from the first.

Proposition 2 is based upon Frigerio's observation [2] that the condition $\left\|\left(T_{t}^{*}-E\right) \psi\right\| \rightarrow 0$, as $t \rightarrow \infty$, for all $\psi \in \mathscr{H}$ is sufficient to ensure that $\| \omega^{\prime} \circ \tau_{t}$ $-\omega^{\prime} \circ M_{\tau} \| \rightarrow 0$ as $t \rightarrow \infty$ for all normal $\omega^{\prime}$. Next we derive a generalization of Frigerio's necessary conditions.

First define the sets

$$
\begin{aligned}
& N(\tau)=\left\{A ; \tau_{t}\left(A^{*} A\right)=\tau_{t}(A)^{*} \tau_{t}(A), t>0\right\}, \\
& N^{+}(\tau)=\left\{A ; \tau_{t}\left(A A^{*}\right)=\tau_{t}(A) \tau_{t}(A)^{*}, t>0\right\}, \\
& \mathcal{N}(\tau)=N(\tau) \cap N^{+}(\tau) .
\end{aligned}
$$

Clearly $\mathscr{N}(\tau)$ is self-adjoint. Moreover if $A \in N(\tau)$ then

$$
\begin{aligned}
\tau_{t+s}\left(A^{*} A\right) & =\tau_{t}\left(\tau_{s}(A)\right)^{*} \tau_{t}\left(\tau_{s}(A)\right) \\
& \leqq \tau_{t}\left(\tau_{s}(A)^{*} \tau_{s}(A)\right) \\
& =\tau_{t}\left(\tau_{s}\left(A^{*} A\right)\right)=\tau_{t+s}\left(A^{*} A\right) .
\end{aligned}
$$

Hence $\tau_{s}(A) \in N(\tau)$ for all $s>0$. Applying a similar argument to $N^{+}(\tau)$ one concludes that $N(\tau), N^{+}(\tau)$, and $\mathscr{N}(\tau)$, are all $\tau$-invariant sets. Next by application of the Cauchy-Schwarz inequality to the form $S_{t}(A, B)=\tau_{t}\left(A^{*} B\right)-\tau_{t}(A)^{*} \tau_{t}(B)$, evaluated in a state, one finds that

$$
N(\tau)=\left\{A ; \tau_{t}(B A)=\tau_{t}(B) \tau_{t}(A), t>0, B \in \mathscr{M}\right\}
$$

Thus $N(\tau)$ is a subspace of $\mathscr{M}$. Similarly $N^{+}(\tau)$ and $\mathscr{N}(\tau)$ are subspaces. But if $A_{1}, A_{2} \in N(\tau)$ then

$$
\begin{aligned}
\tau_{t}\left(B A_{1} A_{2}\right) & =\tau_{t}\left(B A_{1}\right) \tau_{t}\left(A_{2}\right) \\
& =\tau_{t}(B) \tau_{t}\left(A_{1}\right) \tau_{t}\left(A_{2}\right)=\tau_{t}(B) \tau_{t}\left(A_{1} A_{2}\right)
\end{aligned}
$$


and hence $A_{1} A_{2} \in N(\tau)$. Similarly $N^{+}(\tau)$ and $\mathcal{N}(\tau)$ are algebras. Finally if $A_{n} \in N(\tau)$ is a sequence which converges strongly to $A$ then

$$
\begin{aligned}
\omega\left(S_{t}(A, A)\right) & =\lim _{n \rightarrow \infty}\left\{\left(A_{n} \Omega, A_{n} \Omega\right)-\left(A_{n} \Omega, T_{t}^{*} T_{t} A_{n} \Omega\right)\right\} \\
& =\lim _{n \rightarrow \infty} \omega\left(S_{t}\left(A_{n}, A_{n}\right)\right)=0,
\end{aligned}
$$

and by faithfulness $S_{t}(A, A)=0$. Thus the sets $N(\tau), N^{+}(\tau)$, and $\mathscr{N}(\tau)$ are strongly closed. But in Sect. 1 we observed that $\mathscr{M}(\tau) \subseteq \mathscr{N}(\tau)$ and hence $\mathscr{N}(\tau)$ is a $\tau$-invariant $W^{*}$-subalgebra of $\mathscr{M}$ which contains the fixed point algebra $\mathscr{M}(\tau)$.

Next let $\sigma$ denote the restriction of $\tau$ to $\mathscr{N}(\tau)$. Clearly $\sigma$ is $a^{*}$-morphism but it is faithful because if $\sigma_{t}(A)=0$ then

$$
\omega\left(A^{*} A\right)=\omega\left(\sigma_{t}\left(A^{*} A\right)\right)=\omega\left(\sigma_{t}(A)^{*} \sigma_{t}(A)\right)=0,
$$

and this is equivalent to $A=0$. Hence by a standard argument, $\sigma$ extends to a group of *automorphisms of $\mathscr{N}(\tau)$, which is automatically isometric. It follows immediately that $\mathscr{N}(\tau)$ is the largest $\tau$-invariant $W^{*}$-subalgebra of $\mathscr{M}$ on which $\tau$ is equal to a group of *automorphisms.

The following result gives both necessary and sufficient conditions for uniform convergence. Note that $l_{\psi}$ denotes the normal linear functional $l_{\psi}(A)=(\psi, A \Omega)$ and $l_{\psi}$ is positive if, and only if, $\psi \in \overline{\mathscr{M}_{+}^{\prime} \Omega}$.

Theorem 5. Consider the following conditions

1. $\lim _{t \rightarrow \infty}\left(\psi,\left(T_{t} T_{t}^{*}-E\right) \psi\right)=0$,

2. $\lim _{t \rightarrow \infty}\left\|l_{\psi} \circ \tau_{t}-l_{\psi} \circ M_{\tau}\right\|=0$,

3. $(F-E) \psi=0$, where $F=[\mathcal{N}(\tau) \Omega]$.

It follows that $1 \Rightarrow 2 \Rightarrow 3$.

Conversely if $T$ is normal and $\mathscr{M}$ is abelian, or, more generally if $T$ is normal and $T_{t}^{*} \mathscr{M}_{+} \Omega \supset \mathscr{M}_{+} \Omega$ for all $t>0$ then $3 \Rightarrow 1$.

Proof. $1 \Rightarrow 2$. This follows from the straightforward estimate

$$
\left\|l_{\psi}^{\circ} \tau_{t}-l_{\psi} \circ M_{\tau}\right\|^{2} \leqq\left\|\left(T_{t}^{*}-E\right) \psi\right\|^{2}=\left(\psi,\left(T_{t} T_{t}^{*}-E\right) \psi\right) .
$$

$2 \Rightarrow 3$. One has

$$
\begin{aligned}
\left\|l_{\psi} \circ \tau_{t}-l_{\psi} \circ M_{\tau}\right\| & =\sup \left\{\left|l_{\psi}\left(\tau_{t}(A)\right)-l_{\psi}\left(M_{\tau}\left(\tau_{t}(A)\right)\right)\right| ; A \in \mathscr{M},\|A\|=1\right\} \\
& \geqq \sup \left\{\left|l_{\psi}\left(\sigma_{t}(A)\right)-l_{\psi}\left(M_{\tau}\left(\sigma_{t}(A)\right)\right)\right| ; A \in \mathscr{N}(\tau),\|A\|=1\right\} \\
& =\sup \left\{\left|l_{\psi}(A)-l_{\psi}\left(M_{\tau}(A)\right)\right| ; A \in \mathscr{N}(\tau),\|A\|=1\right\} \\
& =\sup \{|(\psi,(\mathbb{1}-E) A \Omega)| ; A \in \mathscr{N}(\tau)\|A\|=1\}
\end{aligned}
$$

where the third step uses the isometric property of $\sigma$. Thus Condition 2 implies that $F(\mathbb{1}-E) \psi=0$. But since $\mathscr{M}(\tau) \subseteq \mathscr{N}(\tau)$ and $E=[\mathscr{M}(\tau) \Omega]$ one has $F E=E$. Hence Condition 3 is valid.

Next note that it follows from [4, Lemmas 2 and 4], $T_{t}^{*} \mathscr{M}_{+}^{\prime} \Omega \subseteq \mathscr{M}_{+}^{\prime} \Omega$ and $T_{t}^{*} \mathscr{M}_{+} \Omega \subseteq \overline{\mathscr{M}_{+} \Omega}$ that $T_{t}^{*} \mathscr{M}_{+} \Omega \supset \mathscr{M}_{+} \Omega$. If, however, $\mathscr{M}$ is abelian $\mathscr{M}=\mathscr{M}^{\prime}$. 
Hence in both cases $T_{t}^{*} \mathscr{M}_{+} \Omega \subseteq \mathscr{M}_{+} \Omega$. Consequently there exists a semigroup of positive, identity preserving, hence contractive, maps $\tau^{*}$ of $\mathscr{M}$ into $\mathscr{M}$ such that

$$
\tau_{t}^{*}(A) \Omega=T_{t}^{*} A \Omega .
$$

The assumed normality of $T$ then ensures that $\tau \tau^{*}=\tau^{*} \tau$, i.e., $\tau$ is normal with respect to $\omega$ in the sense of Frigerio [2]. (The converse is also true; Frigerio's condition implies that $T$ is normal and $T_{t}^{*} \mathscr{M}_{+} \Omega \subseteq \overline{\mathscr{M}_{+} \Omega}$.)

Next consider the converse statement.

$3 \Rightarrow 1$. If $s<t$

$$
\begin{aligned}
\left(A \Omega, T_{t} T_{t}^{*} A \Omega\right) & =\omega\left(\tau_{t}(A)^{*} \tau_{t}(A)\right) \\
& \leqq \omega\left(\tau_{t-s}\left(\tau_{s}(A)^{*} \tau_{s}(A)\right)\right)=\left(A \Omega, T_{s} T_{s}^{*} A \Omega\right),
\end{aligned}
$$

where we have used the normality of $T$. Hence $t \rightarrow T_{t} T_{t}^{*}$ is monotonically decreasing and its strong limit $G$ exists. Since $T$ is normal, $G$ is an orthogonal projection. But for $A \in \mathscr{N}(\tau)$ and $B \in \mathscr{M}$ one has $S_{t}(B, A)=0$ and hence

$$
(B \Omega, A \Omega)=\left(\tau_{t}(B) \Omega, \tau_{t}(A) \Omega\right),
$$

or, equivalently

$$
(B \Omega, A \Omega)=\left(B \Omega, T_{t}^{*} T_{t} A \Omega\right)=\left(B \Omega, T_{t} T_{t}^{*} A \Omega\right) .
$$

It follows immediately that $F=G F$ and hence $G \geqq F \geqq E$.

Next remark that $T_{t} T_{t}^{*} \mathscr{M}_{+} \Omega \subseteq \overline{\mathscr{M}_{+} \Omega}$. Hence $G \mathscr{M}_{+} \Omega \subseteq \overline{\mathscr{M}_{+} \Omega}$ and then $G \mathscr{M}_{+} \Omega \subseteq \mathscr{M}_{+} \Omega$ by [4]. Thus there exists a positive map $g$ of $\mathscr{M}$ into $\mathscr{M}$ such that

for all $A \in \mathscr{M}$. But

$$
g(A) \Omega=G A \Omega
$$

$$
\omega\left(S_{t}(g(A), g(A))\right)=(A \Omega, G A \Omega)-\left(A \Omega, G T_{t} T_{t}^{*} G A \Omega\right)=0,
$$

and since $\omega$ is faithful $g(A) \in N(\tau)$. But conversely if $A \in N(\tau)$ then

$$
\begin{aligned}
H(A-g(A)) \Omega \|^{2} & =(A \Omega, A \Omega)-(A \Omega, G A \Omega) \\
& =\lim _{t \rightarrow \infty} \omega\left(S_{t}(A, A)\right)=0
\end{aligned}
$$

and hence $A=g(A)$. Thus $g$ maps $\mathscr{M}$ onto $N(\tau)$. This implies $N(\tau)=N^{+}(\tau)=\mathscr{N}(\tau)$ and $G \leqq F$. Since we have already established that $F \leqq G$, this gives $F=G$. Consequently Condition 3 implies Condition 1.

Remarks. 1. The equivalence of Condition 2 and 3 is a local version of Frigerio's Theorem 4.2 in [2]. Note that Condition 3 is valid for all $\psi \in \mathscr{H}$ if, and only if, $\mathscr{N}(\tau)$ $=\mathscr{M}(\tau)$.

2. In the foregoing proof we established that $\mathscr{N}(\tau)=N(\tau)=N^{+}(\tau)$ whenever $T$ is normal and $T_{t}^{*} \mathscr{M}_{+} \Omega \subseteq \overline{\mathscr{M}_{+} \Omega}, t>0$.

3. It follows from [4] that $T_{t}^{*} \mathscr{M}_{+} \Omega \subseteq \overline{\mathscr{M}_{+} \Omega}, t>0$, if and only if, $T$ commutes with the modular conjugation $J$ associated with the pair $(\mathscr{M}, \Omega)$, or, alternatively if, and only if, $\tau$ commutes with the modular automorphism group associated with $(\mathscr{M}, \Omega)$. 


\section{The Modulus Semigroup}

Theorem 5 gives a characterization of pointwise uniformly clustering states when $\mathscr{N}(\tau)=\mathscr{M}(\tau)$. In this section we analyze the situation when $\mathscr{N}(\tau) \neq \mathscr{M}(\tau)$.

First note that if $A \in \mathscr{N}(\tau)$ and $B \in \mathscr{M}$ then $S_{t}(A, B)=0$. Applying this relation to $\Omega$ and appealing to cyclicity one deduces that

$$
T_{t} A=\tau_{t}(A) T_{t} .
$$

Therefore

$$
T_{t}^{*} T_{t} A=T_{t}^{*} \tau_{t}(A) T_{t} .
$$

If $A$ is self-adjoint it follows by conjugation that $A$ and $\left|T_{t}\right|^{2}$ commute. Consequently one concludes that $\mathscr{N}(\tau) \cong \mathscr{M} \cap|T|^{\prime}$. Next we analyze conditions under which equality occurs and, moreover, $\mathscr{N}(\tau)$ can be identified as the fixed points of a semigroup, the modulus semigroup.

Throughout the remainder of this section we make the following assumptions, for all $t>0$.

1. $T_{t}^{*} \mathscr{M}_{+} \Omega \subseteq \overline{\mathscr{M}_{+} \Omega}$

2. $T_{t}$ is normal.

It follows, as in the previous section, that there exists a positive identity preserving semigroup $\tau^{*}$ of maps of $\mathscr{M}$ into $\mathscr{M}$ such that

$$
\tau_{t}^{*}(A) \Omega=T_{t}^{*} A \Omega,
$$

and $\tau^{*}$ commutes with $\tau$. We now define the modulus semigroup by

$$
|\tau|_{t}=\tau_{t / 2}^{*} \tau_{t / 2}
$$

and note that

$$
\begin{aligned}
|\tau|_{t}(A) \Omega & =T_{t / 2}^{*} T_{t / 2} A \Omega \\
& =\left|T_{t / 2}\right|^{2} A \Omega=\left|T_{t}\right| A \Omega .
\end{aligned}
$$

Again $|\tau|$ is positive and identity preserving. Moreover it follows from the proof of $3 \Rightarrow 1$ in Theorem 5 that the strong limit

$$
G=\lim _{t \rightarrow \infty}\left|T_{t}\right|
$$

exists and $G=F$ where $F=[\mathcal{N}(\tau) \Omega]$. But the mean $M_{|\tau|}(A)$ of $A$ with respect to the semigroup $|\tau|$ is determined by the relation

$$
M_{|\tau|}(A) \Omega=G A \Omega=F A \Omega .
$$

Thus $R\left(M_{|\tau|}\right)=\mathscr{N}(\tau)$ and $\mathscr{N}(\tau)$ is the fixed point set of $|\tau|$, i.e., $\mathscr{N}(\tau)=\mathscr{M}(|\tau|)$. But if $A \in \mathscr{M} \cap G^{\prime}$ then

$$
A \Omega=G A \Omega=F A \Omega
$$

and hence $A \in \mathscr{N}(\tau)$. Combining these conclusions one has

$$
\mathscr{N}(\tau)=\mathscr{M} \cap|T|^{\prime}=\mathscr{M} \cap F^{\prime} .
$$

Next it is tempting to apply the results of Sects. 2,3 , and 4 , to the pair $(\mathscr{M},|\tau|)$. The only problem is that it is not evident that $|\tau|$ satisfies the condition of strong positivity. To circumvent this difficulty we assume that $\tau$ is 2-positive. By this we 
mean that $\tau_{t}\left(A_{i}^{*} A_{j}\right), i, j=1,2$, is a positive $\mathscr{M}$-valued $2 \times 2$ matrix for all $t>0$. We recall that a 2-positive semigroup is automatically strongly positive [7] (but there exist strongly positive operators which are not 2-positive [8]).

Proposition 3. If $\tau$ is 2-positive then $\tau^{*}$ is 2-positive and $|\tau|$ is strongly positive.

Proof. Since $\Omega$ is cyclic for $\mathscr{M}^{\prime}$ the 2-positivity of $\tau$ is equivalent to

$$
\sum_{i=1}^{2} \sum_{j=1}^{2}\left(\Omega, B_{i}^{\prime *} \tau_{t}\left(A_{i}^{*} A_{j}\right) B_{j}^{\prime} \Omega\right) \geqq 0
$$

for all $t>0, A_{i}, A_{j} \in \mathscr{M}$, and $B_{i}^{\prime}, B_{j}^{\prime} \in \mathscr{M}^{\prime}$. This can be reexpressed as

$$
\sum_{i=1}^{2} \sum_{j=1}^{2}\left(\Omega, B_{i}^{\prime *} B_{j}^{\prime} T_{t} A_{i}^{*} A_{j} \Omega\right) \geqq 0 .
$$

Now if $A \in \mathscr{M}, B^{\prime} \in \mathscr{M}^{\prime}$, and $J$ is the modular conjugation, then $A^{\prime}=J A J \in \mathscr{M}^{\prime}$ and $B=J B^{\prime} J \in \mathscr{M}$. Moreover $J$ commutes with $T$ by [4]. Thus

$$
\begin{aligned}
\left(\Omega, B^{\prime} T_{t} A \Omega\right) & =\left(\Omega, J B J T_{t} J A^{\prime} J \Omega\right) \\
& =\left(\Omega, J B T_{t} A^{\prime} \Omega\right) \\
& =\left(B T_{t} A^{\prime} \Omega, \Omega\right)=\left(\Omega, A^{\prime *} T_{t}^{*} B^{*} \Omega\right) .
\end{aligned}
$$

The 2-positivity of $\tau^{*}$ follows immediately from combination of these observations. Consequently $\tau *$ is strongly positive and $|\tau|$ is strongly positive, because it is the product of the two strongly positive maps $\tau$ and $\tau^{*}$.

Thus if $\tau$ is 2-positive one can apply the results of the previous sections to the pair $(\mathscr{M},|\tau|)$. Since the $\tau$-invariant state $\omega$ is automatically $|\tau|$-invariant one concludes that Theorems $1-3$, are valid with the replacements $\tau \rightarrow|\tau|, E \rightarrow F$, $\mathscr{M}(\tau) \rightarrow \mathscr{N}(\tau)$.

It is of some interest to examine the condition of $\mathbb{R}_{+}$-abelianness of $(\mathscr{M},|\tau|, \omega)$ in more detail. This condition, which ensures the uniqueness of the barycentric decomposition of $\omega$ into $|\tau|$-ergodic states can be written

$$
\begin{aligned}
0=[F A F, F B F] & =\lim _{\alpha} \sum_{i} \lambda_{i}^{\alpha}\left\{F A T_{t_{i}}^{*} T_{t_{i}} B F-F B T_{t_{i}}^{*} T_{t_{i}} A F\right\} \\
& =\lim _{\alpha} \sum_{i} \lambda_{i}^{\alpha} \mathrm{T}_{t_{i}}^{*} \mathrm{~F}\left[\tau_{t_{i}}(\mathrm{~A}), \tau_{t_{i}}(\mathrm{~B})\right] \mathrm{FT}_{t_{i}},
\end{aligned}
$$

where the limit is over the net of finite convex combinations. [Here we have used the identity $T_{t} A F=\tau_{t}(A) F T_{t}$ which follows from noting that $S_{t}(A, B) \Omega=0$ for all $A \in \mathscr{M}$ and $B \in \mathscr{N}(\tau)$.] In particular it is necessary that

$$
\lim _{\alpha} \sum_{i} \lambda_{i}^{\alpha} E\left[\tau_{t_{i}}(A), \tau_{t_{i}}(B)\right] E=0
$$

for all $A, B \in \mathscr{M}$. This should be contrasted with the condition

$$
\lim _{\alpha} \sum_{i} \lambda_{i}^{\alpha} E\left[\tau_{t_{i}}(A), B\right] E=0
$$

which is both necessary and sufficient to ensure that $\omega$ has a unique barycentric decomposition into $\tau$-ergodic states. Both these conditions reflect a form of 
asymptotic abelianness but $(*)$ could be described as a "local dissipation" condition whilst $\left(*^{*}\right)$ is a property of escape to infinity.

Finally the $\tau$-invariant, $|\tau|$-ergodic states can be characterized by pointwise uniform clustering with respect to $\tau$.

Theorem 6. If $\tau$ is 2-positive it follows that

$$
\lim _{t \rightarrow \infty}\left\|\omega^{\prime} \circ|\tau|_{t}-\omega^{\prime} \circ M_{|\tau|}\right\|=0,
$$

for all normal states $\omega^{\prime}$ of the $\tau$-invariant state $\omega$. Hence the following conditions are equivalent;

1. $\omega$ is $|\tau|$-ergodic,

2. $\lim _{t \rightarrow \infty} \omega\left(\tau_{t}(A) \tau_{t}(B)\right)=\omega(A) \omega(B)$ for all $A, B \in \mathscr{M}$,

3. $\lim \left\|\omega^{\prime} \circ \tau_{t}-\omega\right\|=0$ for all normal states $\omega^{\prime}$.

Proof. Since $|\tau|$ is determined by the self-adjoint contraction semigroup $t \rightarrow\left|T_{t}\right|$ the first statement follows from Proposition 1 applied to $|\tau|$.

Next consider the equivalences between the three conditions $1 \Leftrightarrow 2$. Since

$$
\omega\left(\tau_{t}(A) \tau_{t}(B)\right)=\left(\Omega, A T_{t}^{*} T_{t} B \Omega\right),
$$

Condition 2 is equivalent to $F=E_{\Omega}$. But this is equivalent to Condition 1 by Theorem 3, applied to $|\tau|$.

$1 \Leftrightarrow 3$. Condition 1 is equivalent to

$$
\lim _{t \rightarrow \infty}\left\|\omega^{\prime} \circ|\tau|_{t}-\omega\right\|=0
$$

for all normal states by Proposition 2 applied to $|\tau|$. But this implies that $\omega^{\prime} \circ M_{|\tau|}$ $=\omega$ for all $\omega^{\prime}$ and this is equivalent to $F=E_{\Omega}$. This is in turn equivalent to Condition 3 by Theorem 5 .

Davies [9] has emphasized the significance of pointwise uniform clustering in the context of "return to equilibrium" and given specific examples.

\section{Concluding Remarks}

The foregoing results do not require any assumption of continuity of $\tau$ or $T$ as function of $t$. If, however, $\tau$ is weak* continuous then $T$ is weakly, hence strongly, continuous and the results based upon the mean ergodic theorem can be strengthened. In the absence of continuity we defined mean values of $T$ and elements $\tau(A)$ as infinima over convex combinations, but in the continuous case one can use any of the usual averaging processes, Abel means, Cesaro means, etc.

Next we remark that although Theorem 2 gives necessary and sufficient conditions for the existence of a unique maximal measure on $E_{\mathscr{M}}^{\tau}$ with barycentre $\omega$, these conditions do not ensure that the measure is subcentral, i.e., that the abelian subalgebra of $\mathscr{M}^{\prime}$ which corresponds to $\mu$ is contained in the centre $\mathscr{Z}=\mathscr{M} \cap \mathscr{M}^{\prime}$ of $\mathscr{M}$. It is evident that $\mu$ is subcentral if, and only if,

$$
\mathscr{M}(\tau) \cong \mathscr{Z} \cap E^{\prime}
$$


and this inclusion is certainly assured by a suitable hypothesis of asymptotic abelianness. If, however, $\tau$ is replaced by a group of *-automorphism then the equality

$$
\mathscr{M}(\tau)=\mathscr{Z} \cap E^{\prime}
$$

can in fact be characterized by an asymptotic abelianness property, $G$-centrality, (see [1, Chap. 4]). It is unclear whether a similar characterization is true in the semigroup setting.

Finally we note that the assumption of normality of $T$ is a weakness of the results in Sect. 4. If one drops this hypothesis one can deduce, nevertheless, that $\mathscr{N}(\tau)=\mathscr{M} \cap|T|^{\prime}=\mathscr{M} \cap G^{\prime}$, where $G$ is now the largest orthogonal projection such that $G \leqq\left|T_{t}\right|$ for all $t$. It is difficult, however, to identity $\mathscr{N}(\tau)$ as the fixed point set of a positive semigroup. The natural candidate would of course be given by $\hat{\tau}_{t}$ $=\lim \left(\tau_{t / n}^{*} \tau_{t / n}\right)^{n}$, if this limit exists.

Acknowledgements. The author would like to thank R. Streater for his hospitality at Bedford College and to thank B. Davies for numerous illuminating conversations on strongly positive semigroups. The author is also indebted to the British Science Research Council for partial support whilst this work was being carried out.

\section{References}

1. Bratteli, O., Robinson, D.W.: Operator algebras and quantum statistical mechanics, Vol. I. Berlin, Heidelberg, New York: Springer 1979

2. Frigerio, A. : Stationary states of quantum dynamical semigroups. Commun. Math. Phys. 63, 269 276 (1978)

3. Evans, D.E. : Irreducible quantum dynamical semigroups. Commun. Math. Phys. 54, 293-297 (1977)

4. Bratteli, O., Robinson, D.W.: Unbounded derivations of von Neumann algebras. Ann. Inst. H. Poincaré 25 (A), 139-164 (1976)

5. Majewski, A., Robinson, D.W.: Strictly positive and strongly positive semigroups. University of New South Wales Preprint (to be published in the Australian Journal of Mathematics)

6. Radin, C. : Non-commutative mean ergodic theory. Commun. Math. Phys. 21, 291-302 (1971)

7. Choi, M.-D.: A Schwarz inequality for positive linear maps on $C^{*}$-algebras. Ill. J. Math. 18, 565-574 (1974)

8. Choi, M.-D.: Inequalities for positive linear maps. J. Operat. Theory 4, 271-285 (1980)

9. Davies, E.B.: Irreversible dynamics of infinite fermion systems. Commun. Math. Phys. 55, 231-258 (1977)

Communicated by R. Jost

Received May 5, 1981; in revised form October 5, 1981 